\title{
Perceived depth modulates perceptual resolution
}

\author{
Tasfia Ahsan ${ }^{\mathrm{a}, \mathrm{b}}$, Kathryn Bolton ${ }^{\mathrm{a}}$, Laurie M. Wilcox ${ }^{\mathrm{a}, \mathrm{b}}$, Erez Freud $^{\mathrm{a}, \mathrm{b}}$ \\ a Department of Psychology, York University, Toronto, Ontario M3J 1P3, Canada \\ b Centre for Vision Research, York University, Toronto, Ontario M3J 1P3, Canada
}

Acknowledgements - This study was supported by the Vision Science to Applications (VISTA) program funded by the Canada First Research Excellence Fund (CFREF, 2016-2023) and by the Discovery grant from the Natural Sciences and Engineering Research Council (NSERC).

We thank Johnathan Tong for his guidance in data analysis and Krista Chiasson for her help in data collection.

The authors declare no conflicts of interest.

Correspondence concerning this article should be addressed to Erez Freud (efreud@yorku.ca) 


\begin{abstract}
Humans constantly use depth information to support perceptual decisions about object size and location in space, as well as planning and executing actions. It was recently reported that perceived depth modulates perceptual performance even when depth information is not relevant to the task, with faster shape discrimination for objects perceived as being close to the observer. However, it is yet to be determined if the observed "close advantage" reflects differences in psychophysical sensitivity or response bias. Moreover, it is unclear whether this advantage is generalizable to other viewing situations and tasks. To address these outstanding issues, we evaluated whether visual resolution is modulated by perceived depth defined by 2D pictorial cues. In a series of experiments, we used the method of constant stimuli to measure the precision of perceptual judgements for stimuli positioned at close, far, and flat perceived distances. In Experiment 1, we found that size discrimination was more precise when the object was perceived to be closer to the observers. Experiments $2 \mathrm{a}$ and $2 \mathrm{~b}$ extended this finding to a visual property orthogonal to depth information, by showing superior orientation discrimination for "close" objects. Finally, Experiment 3 demonstrated that the close advantage also occurs when performing high-level perceptual tasks such as face perception. Taken together, our results provide novel evidence that the perceived depth of an object, as defined by pictorial cues, modulates the precision of visual processing for close objects.
\end{abstract}

Keywords: Depth perception, JND, close advantage, constant stimuli, object recognition 


\section{Introduction}

The ability to see space in three dimensions (3D) is a fundamental achievement of the human visual system. Even though each eye receives a flat two-dimensional (2D) image, the human visual system extracts information from a set of monocular and binocular depth cues to provide us with the 3D layout of the visual environment (Howard, 2012). This information is critical for the perception of relative depth and distance of objects, but also for guidance of actions and interactions with our surroundings.

The importance of depth information to everyday life is also demonstrated by results that show that viewing distance modulates sensory processing. Based on these findings it has been suggested that 3D space can be sub-divided relative to the distance from the observer. Peripersonal space (PPS) is a region of space that immediately surrounds our body, while extrapersonal space (EPS) is a region that falls beyond our body's reach (Previc, 1990, 1998; Rizzolatti et al., 1997). Notably, there is an accumulation of evidence pointing to a privileged processing of objects within PPS (Kaas \& Mier, 2006; Làdavas, 2002). For example, participants were found to be more accurate when performing a simple spatial discrimination task for objects in PPS than in EPS (Dufour \& Touzalin, 2008). Along similar lines, participants showed enhanced change detection abilities when they positioned their hands closer to a monitor on which the stimuli were displayed (Tseng \& Bridgeman, 2011).

Both the above-mentioned studies (Tseng \& Bridgeman, 2011; Dufour \& Touzalin, 2008) suggest that perceptual and attentional performances are affected by the location of the hand in PPS. As such, this privileged processing may reflect top-down processes, such as the relationship between affordance and space. According to this view, the enhanced processing is triggered by specific object features (e.g., handles), particularly when they fall within the reachable space 
(Costantini et al., 2010). This link between affordance and PPS is corroborated by other behavioral studies showing that participants recognize functional/manipulation verbs more rapidly when objects are placed in PPS (Costantini et al., 2011), as well as neurophysiological studies showing the existence of bimodal neurons (that respond to both visual and tactile stimuli) responsible for coding visual PPS (Làdavas, 2002).

While the preceding studies attributed the privileged processing within PPS to action related mechanisms, a recent study by Blini and colleagues (2018) suggests that even shape perception is enhanced when objects are perceived as close to the observer and that this effect holds even when depth is defined by pictorial cues. In their study, response times in a 2-AFC object classification task decreased as the perceived distance of the target decreased. However, since the effect is evident only in the reaction time measure and the task was a binary one (rather than a continuous transformation), it is not clear whether the close advantage effect reflects changes in response bias or an enhancement of perceptual resolution. Importantly, it was recently reported that humans are less able to inhibit motor actions directed to rewarding cues that are within reach, further demonstrating the potential role of response bias in the close advantage effect (O'Connor et al., 2021). Thus, it is important that we determine if this phenomenon is apparent in measures of psychophysical sensitivity (i.e., Just Noticeable Difference, JND) and not only in terms of reaction time.

To address these gaps, the current study explores whether visual resolution is modulated by perceived depth across a range of tasks and visual attributes, using the method of constant stimuli (Urban, 1910) that allows detailed characterization of perceptual sensitivity. We used 2D pictorial cues of the Ponzo illusion (Fig. 1A) to induce changes in perceived depth. A large number of studies have established that the Ponzo illusion reliably induces significant depth 
percepts across ages (Freud et al., 2021; Gandhi et al., 2015) and species (Timney \& Keil, 1996; Gunderson et al., 1993).

In Experiment 1, we examined the effects of this manipulation on size discrimination. However, given the inherent relationship between size and depth perception, in Experiment 2a, we explored whether sensitivity to orientation, a task orthogonal to depth processing, is similarly affected by perceived depth. In a follow up Experiment 2b, we replicated the orientation task while addressing a potential confound due to the relative sizes of the background surfaces.

Finally, in Experiment 3, we examined whether higher-level visual properties were also impacted by perceived depth using a face identification task. Collectively these experiments provide compelling evidence that the visual system prioritizes processing of objects perceived as close to us.

\section{Experiment 1: Size}

\section{Methods}

\section{Data Availability}

The datasets generated for all experiments are available in the OSF repository: https://osf.io/974du/

\section{Participants}

Data was obtained from 18 healthy adults (age: $\mathrm{M}=23.8, \mathrm{SD}=9.26 ; 2$ males), none of whom participated in any of the other experiments reported here. The sample size used for this experiment was consistent with the average sample size used in similar studies (Ganel et al., 2008; Blini et al., 2018). The data from two participants were excluded because of near chance 
level performance for all conditions (indicative of guessing). All participants were recruited from York University's undergraduate research participant pool (URPP) and received course credit for their participation. Participants were screened to be right-handed with normal to corrected vision and were enrolled in the study after obtaining their informed written consent form. All experiments were approved by the York University Human Participants Review Committee (HPRC) prior to data collection.

\section{Materials and Apparatus}

Experiment 1 was conducted in a laboratory setting using a PC desktop computer with Windows 10 operating system. The stimuli were displayed on a 24-inch monitor with a viewing distance of $50 \mathrm{~cm}$, the resolution of the monitor was 1900 x 1200 pixels. Experimental stimuli were drawn and displayed using PsychoPy3 (Peirce, 2007). All lines subtended a visual angle of $2.48^{\circ} \times 2.98^{\circ}$. The main experiment consisted of 12 levels, a step size of $0.07 \mathrm{~cm}\left(0.05^{\circ}\right.$ change in visual angle), with the conditions centred on the reference height of $2.00 \mathrm{~cm}$ (See Fig. 1B for sample length continua).

On each trial, two lines ${ }^{1}$ of different heights were overlaid on a version of the Ponzo Illusion or non-illusory (Ponzo Flat) background (Fig. 1A). Both versions of the Ponzo background were adopted from previous experiments (Ganel et al., 2008; Freud et al., 2021). Stimuli placed on the larger rectangle of the Ponzo Illusion appeared "close" to the observer, while stimuli placed on the smaller rectangle appeared "far" from the observer. The Ponzo "flat" condition was used as a control and did not contain any depth cues. The size of both background

\footnotetext{
1 The stimuli in Experiment 1 are better characterized as elongated rectangles than "lines". However, we refer to these stimuli as lines in order to avoid confusion when referring to the rectangles that form the Ponzo background.
} 
was $23.85 \mathrm{~cm} \times 11.36 \mathrm{~cm}$ (resulting in a visual angle of $28.94^{\circ} \times 14.02^{\circ}$ ), which were counterbalanced between left and right for the two perceived depths (close and far) and no depth (flat) conditions. All stimuli were presented at the same height relative to a fixation cross to rule out potential confounds due to relative height in the field. Randomized interleaved conditions were used to show all stimuli across the three depth conditions.

\section{Procedure}

Each trial consisted of a fixation phase $(800 \mathrm{~ms})$ followed by the presentation of a pair of lines randomly chosen to be in the close, flat, or far portion of the Ponzo backgrounds using a method of constant stimuli. On each trial, participants were asked to determine which of the two lines was longer. They indicated their responses by pressing $\mathrm{F}$ (for left) and $\mathrm{K}$ (for right) keys on the keyboard. Stimuli were presented for up to a maximum of $3000 \mathrm{~ms}$ and were replaced by the next stimuli once participants had made a response. If participants failed to respond within the given time, then the stimuli were replaced by a black screen until a response was made. Participants were asked to respond as quickly and accurately as possible. A total of 72 trials (12 sizes $\mathrm{x} 3$ depths $\mathrm{x} 2$ sides (left and right)) were repeated 12 times resulting in a total of 864 trials; therefore, each size level was presented a total of 24 times (12 sizes x 2 counterbalanced sides) in a randomized order. The experiment duration was approximately 30 minutes with a break at the mid-point.

\section{Data analysis}

Statistical analyses were conducted using JASP (JASP team, 2018), R (R Core Team, 2020), and MATLAB (R2018b, Mathworks). The proportion of 'longer' responses, and reaction 
times (RT), reported as milliseconds, were recorded for each participant. Just noticeable difference (JND) values were calculated for each observer by averaging the proportion 'longer' responses for each condition and fitting a cumulative normal psychometric function using the maximum likelihood method. The JND represents the change in sensitivity to one increment change in stimulus magnitude, thus a smaller JND indicates greater precision, and a shorter RT represents more rapid classification.

\section{Results}

To examine whether perceptual resolution is modulated by the perceived location in depth of the objects, participants discriminated between two lines presented on close vs. far perceived depth planes. The results of Experiment 1 (Fig. 1C) revealed that both JNDs and RTs were lower for objects that were perceived as closer to the observer.

This observation was supported by a repeated measures ANOVA on JND scores with perceived depth as the independent variable. The ANOVA revealed a main effect of perceived depth on size JND $\left[\mathrm{F}_{(2,30)}=7.451, \mathrm{p}<.005, \eta_{\mathrm{p}}{ }^{2}=0.33\right]$. Planned comparisons showed that this effect reflected smaller JNDs for the close condition compared with the far condition $\left(\mathrm{t}_{(15)}=\right.$ 3.36, $\mathrm{p}=0.004$; Mean Difference: 0.100 [CI: $0.036,0.164]$ ); as well as for the flat condition compared with the far condition $\left(t_{15)}=2.54, p=0.02\right.$, Mean Difference $=0.062$ [CI: 0.010 , $0.113])$. The close and flat JND scores were not significantly different from each other $\left(t_{(15)}<1\right.$; Mean Difference $=0.039$ [CI: 0.09, -0.012$]$ ).

The RT results were also consistent with our hypothesis. That is, a main effect of perceived depth was found for size $\mathrm{RT}\left[\mathrm{F}_{(2,30)}=5.84, \mathrm{p}<.01, \eta_{\mathrm{p}}{ }^{2}=0.28\right]$. Results of planned comparisons showed faster RTs for the close condition compared with the far condition $\left(\mathrm{t}_{115)}=\right.$ 
$3.80, \mathrm{p}=0.001 ;$ Mean Difference $=99[\mathrm{CI}: 43,154])$; as well as for the flat condition compared with the far condition $\left(\mathrm{t}_{(15)}=2.54, \mathrm{p}=0.02\right.$ Mean Difference $=102$ [CI: 16.7, 187]). Similar to the JND scores, RTs were similar between the close and flat conditions $\left(\mathrm{t}_{(15)}<1\right.$; Mean Difference $=3$ [CI: $-70,76.4])$.

Together, the results of Experiment 1 revealed a clear effect of perceived depth; both perceptual resolution and speed of judgment improved when objects were positioned on the perceptually closer surface, even though their retinal size remained constant across depth conditions. 

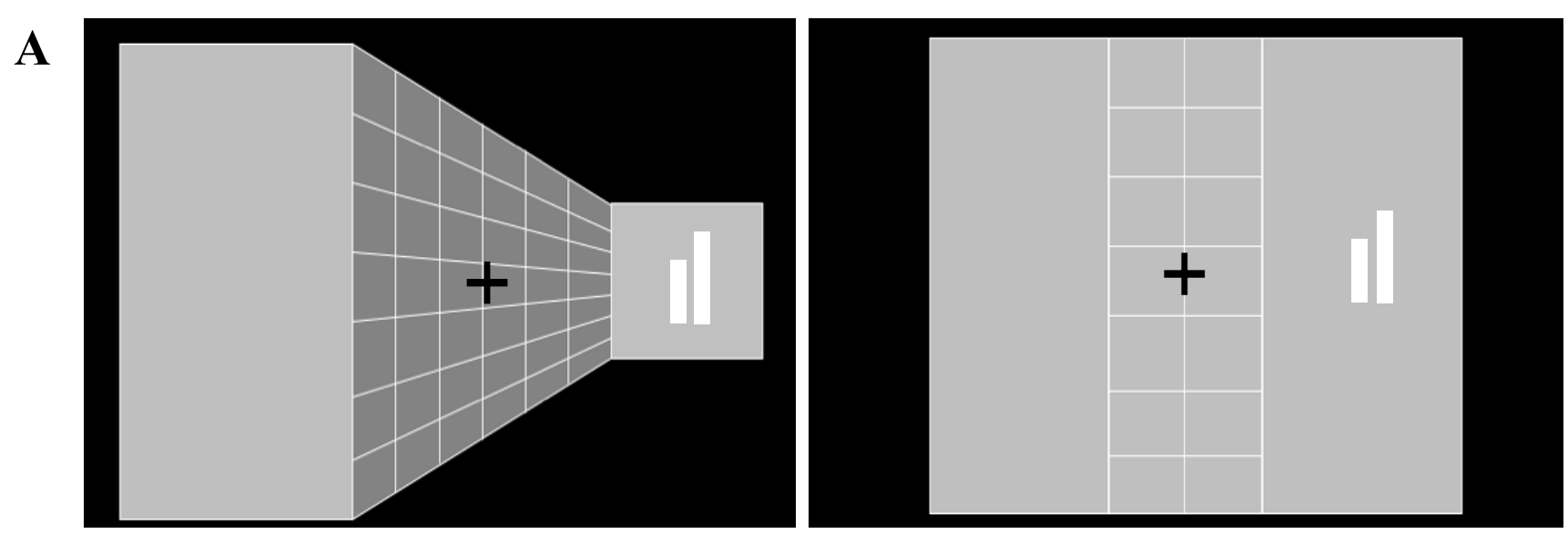

B
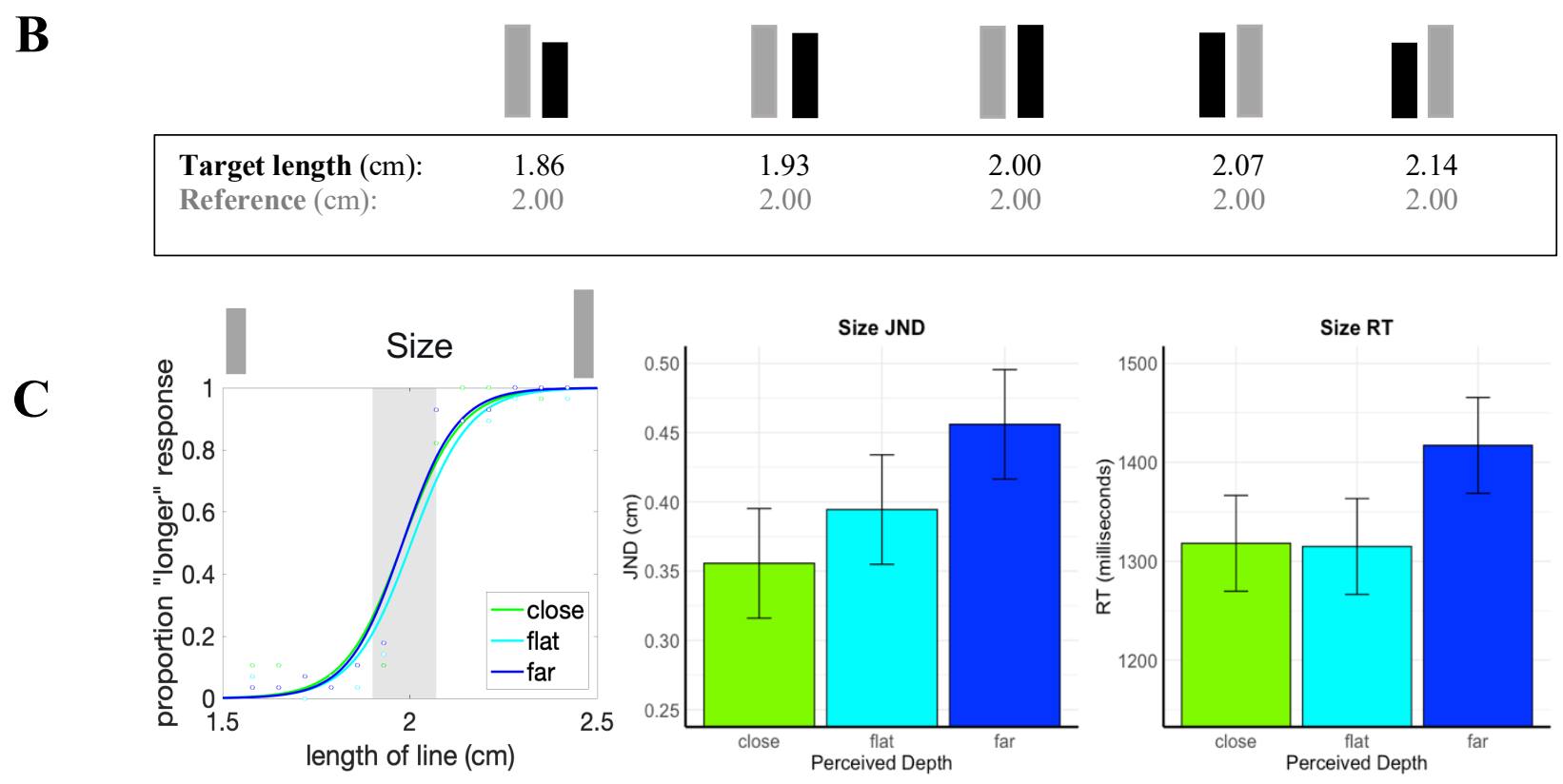

Fig. 1 A) Experimental backgrounds - Ponzo illusion (left) and control "flat" background (right) were used as the background across the different experiments. The Ponzo illusion is based on 2D perspective cues which make stimuli appear "close" or "far" from participant. The Ponzo Flat has all perspective lines removed to provide a non-illusory control background. Sample white lines are used to show how stimuli would be presented on top of the background (example is presented on "far" right side and "flat" right side. B) Experimental stimuli for Experiment 1 - the length of the target lines was manipulated with a step size of $0.07 \mathrm{~cm}$, while the length of the reference line was set at $2.00 \mathrm{~cm}$. C) Results of Experiment 1 - changes in perceptual resolution as a function of perceived depth. Left panel shows the psychometric fit for one representative participant, middle panel shows the average JNDs and right panel shows average RTs. Error bars across all figures are 95\% Confidence Intervals (Jarmasz \& Hollands, 2009). 
accordance with Weber's law (Baird \& Noma, 1978), better perceptual resolution is predicted for smaller objects within the limits of visual acuity. Thus, it could be argued that the results of Experiment 1 simply reflected an adherence to Weber's law based on the perceived object size. To address this concern, in Experiment 2a, we evaluated whether a similar "close advantage" is observed in a task involving orientation classification, a visual feature that should not be modulated by perceived depth and should not be affected by Weber's law.

\section{Method}

\section{Participants}

Data was obtained from 18 adults (age: $\mathrm{M}=23.4, \mathrm{SD}=4.03 ; 4$ males), none of whom participated in any of the other experiments. The data from 3 participants were excluded because of near chance level performance in all conditions. All other aspects of the recruiting process followed the same guidelines as Experiment 1.

\section{Material and Apparatus}

The apparatus and stimuli were same as Experiment 1, with the exception of the following changes. Both lines were $2 \mathrm{~cm}$ in length and $0.2 \mathrm{~cm}$ in width, separated by a distance of $2 \mathrm{~cm}$. The main experiment consisted of 11 levels, step size varying from $0.5^{\circ}$ to $2.5^{\circ}$ rotated clockwise from a vertical position, with the conditions centred on the reference orientation of $15^{\circ}$ (See Fig. 1A for background and Fig. 2A for sample orientation continua).

\section{Procedure}


The procedure was the same as that described in Experiment 1, except that observers were asked to judge line orientations. Participants performed an orientation task involving two lines of different orientations, and determine which line is more rotated clockwise. Each participant underwent a brief pretest consisting of 24 trials repeated four times (total 96 trials). The pretest was used to calculate the step size for each individual and was not included in the final analysis. For the main experiment, a total of 66 trials (11 orientations $\mathrm{x} 3$ depths $\mathrm{x} 2$ counterbalanced sides) were repeated 12 times (total 792 trials); therefore, each level was presented 22 times (11 orientations x 2 counterbalanced sides) in a randomized order.

\section{Results}

In Experiment 2a, participants judged line orientation while their perceived depth was manipulated. As presented in Fig. 2B, in agreement with Experiment 1, more precise orientation classification was found for objects positioned on the surface that appeared to be closer to the observer.

Results of a repeated measures ANOVA on the JND data revealed a main effect of perceived depth $\left[\mathrm{F}_{(2,28)}=3.95, \mathrm{p}<.05, \eta_{\mathrm{p}}{ }^{2}=0.22\right]$. Planned comparisons showed significantly smaller JND scores for the close condition compared to the far condition $\left(\mathrm{t}_{(14)}=2.19, \mathrm{p}=0.004\right.$; Mean Difference $=0.180,[\mathrm{CI}: 0.004,0.355])$ and significantly smaller JNDs for the close condition compared to the flat condition $\left(\mathrm{t}_{(14)}=2.36, \mathrm{p}=0.02\right.$. Mean Difference $=0.205$, $[\mathrm{CI}$ : 0.018, 0.390]). No significant differences were found between far and flat condition JND values $\left(\mathrm{t}_{(14)}<1\right.$; Mean Difference $\left.=-0.025,[\mathrm{CI}:-0.171,0.121]\right)$.

Similarly, a main effect of perceived depth on $\mathrm{RT}$ was found $\left[\mathrm{F}_{(2,28)}=5.65, \mathrm{p}<.01\right.$, $\left.\eta_{\mathrm{p}}{ }^{2}=.28\right]$. Planned pairwise comparisons supported the pattern of results seen in the JND scores 
such that participants performed significantly faster for objects on the close surface compared to both the far $\left(\mathrm{t}_{(14)}=2.92, \mathrm{p}=0.01\right.$, Mean Difference $\left.=69,[\mathrm{CI}: 18,118]\right)$ and the flat condition $\left(\mathrm{t}_{(14)}=2.69, \mathrm{p}=0.01\right.$, Mean Difference $\left.=49,[\mathrm{CI}: 10,88]\right)$. No difference was found between far and flat RTs $\left(\mathrm{t}_{(14)}<1\right.$; Mean Difference $=-19$, [CI: -25, 64]).

Taken together, the results of Experiment 2a support the conclusion of Experiment 1 and provide additional evidence for superior perceptual resolution for objects that appeared to be closer to the observer even for a task which is considered to be independent of depth processing. 
A

\begin{tabular}{|llllll|}
\hline & &
\end{tabular}

B
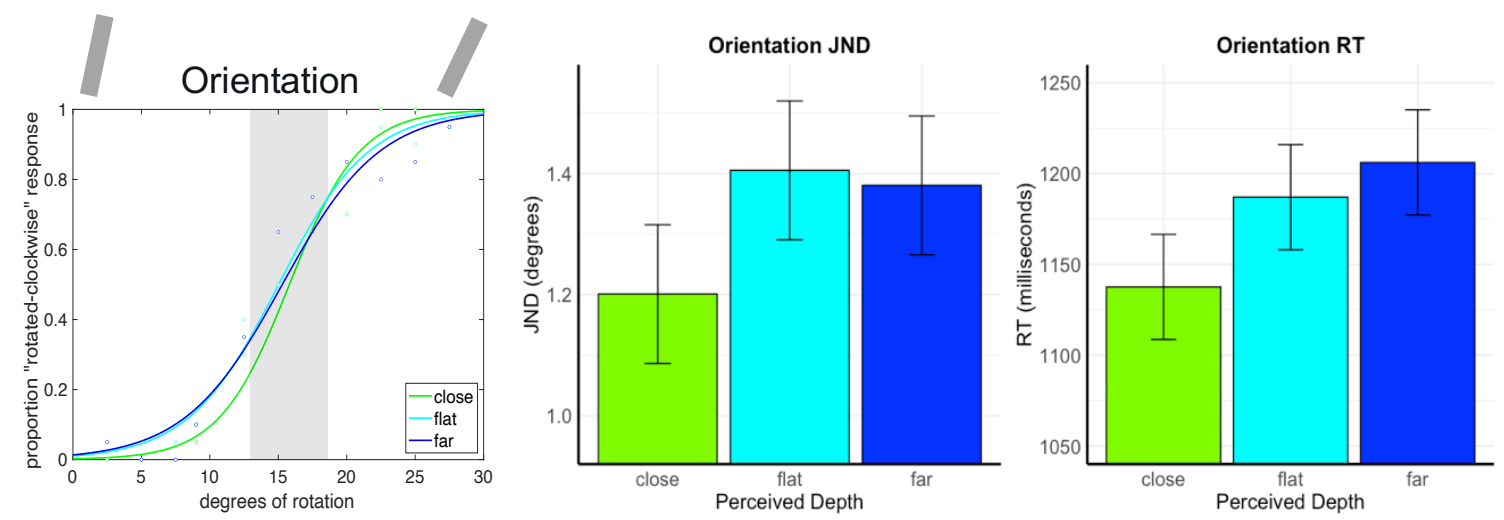

Fig. 2 Experiment 2a - Stimuli and Results A) Experimental stimuli - the orientation of the target line was manipulated with step sizes varying from $0.5^{\circ}$ to $2.5^{\circ}$, rotated clockwise from a vertical position. The orientation of the reference line was set at $15^{\circ}$. During the experiment, the color of the two lines were identical. B) Results - changes in perceptual resolution as a function of perceived depth. Left panel shows the psychometric fit for one representative participant, middle panel shows the average JNDs, and the right panel shows average RTs . 


\section{Experiment 2b: Orientation - controlling for changes in surface size}

The results of Experiment 2a showed that the "close advantage" holds for a visual task unrelated to depth processing. However, an alternative explanation for the observed results relates to the size of the stimuli relative to the sizes of the close and far surfaces. Particularly, the far surface is smaller than the close surface (see Fig. 1A). Since the physical sizes of the target stimuli were constant, the target lines were always closer to the edges of the surface in the far condition compared with the close condition. While unlikely, the proximity of the stimuli to the surface edges may have made it more difficult for the observer to judge the relative orientation of the two test stimuli. Since the surfaces' size manipulation was integral to the 2D depth manipulation, we cannot remove this aspect of the stimuli. However, to evaluate whether the proximity to the surface edges had any impact on performance in this study, we systematically manipulated the surface size in the flat condition. That is, we created two flat conditions: one with large surfaces and the other with small surfaces (Fig. 3A) and examined whether orientation sensitivity differed as a function of surface size (and therefore proximity of the test lines to the surface edges). We also used this experiment as an opportunity to replicate and generalize the results of Experiment 2a.

\section{Method}

Participants:

Due to restrictions on in-person data collection, this experiment was conducted using an online platform. This testing approach also allowed us to access a very broad sample of the population. Notably, we implemented several changes to adapt to this online testing environment. Based on previous studies on efficient online study designs (Mason \& Suri, 2012; 
Reips, 2000), a larger sample size and shorter experiment time was used. In particular, we generated three shorter versions of experiment $2 \mathrm{a}$ and recruited a total of 77 participants across all versions. Sixteen participants were excluded from the results because they failed to follow experimental instructions properly (i.e., guessing) and one participant was excluded because his JND scores deviated from the mean JNDs by more than four standard deviations. Thus, the final analysis was based on a total of 60 participants (20 for each version) (age: $\mathrm{M}=27.8, \mathrm{SD}=6.03$; 21 males).

\section{Material and Apparatus}

Participants were recruited from https://www.prolific.co/ participant pool (Palan \& Schitter, 2018) and received monetary compensation for their participation. Participants were screened to be right-handed with normal to corrected vision and were enrolled in the study after obtaining their informed written consent form through the Qualtrics survey platform (https://www.qualtrics.com/). The orientation task was hosted on the Pavlovia server (https://pavlovia.org/), which offers an online implementation of PsychoPy (Peirce et. al, 2019).

Experimental stimuli and settings were the same as Experiment 2a with the following exceptions. The Ponzo Flat background (Fig. 1A) was replaced with two relative size matched flat conditions (small-flat and big-flat) (see Fig. 3A). Therefore, participants were presented with 4 conditions (far, close, flat-small, flat-big) where all stimuli presentations were counterbalanced for presentation side (left vs. right). Participants performed one of three shorter versions of the experiment, each composed of 9 levels, step size of $3^{\circ}$, centred on the reference orientation of $15^{\circ}$ (See Fig. 3B for sample orientation continua). Version 1 of the experiment contained orientations ranging from $2^{\circ}$ to $26^{\circ}$, Version 2 ranged from $3^{\circ}$ to $27^{\circ}$, and Version 3 from $4^{\circ}$ to $28^{\circ}$. 
Recruitment for each version was independent; participants completing one version did not take part in any other versions of the experiment. Together, the three experiments covered the full experimental range used in Experiment 2a.

\section{Procedure}

Participants began the experiment by performing a set of practice trials with feedback. The practice trials consisted of 8 trials, ranging from orientations $5^{\circ}$ to $25^{\circ}$ with a step size of 2.5. Six consecutive correct answers were required to move on to the main experiment. Once participants successfully passed the practice trials, the main experiment consisted of 72 trials (9 orientations x 4 conditions x 2 counterbalanced sides) repeated 5 times (total 360), so each level was presented a total of 18 times ( 9 orientations $\mathrm{x} 2$ counterbalanced sides). The experiment duration was approximately 10 minutes without any breaks.

\section{Results}

To rule out relative size as a potential confound, we replicated the orientation task of Experiment $2 \mathrm{a}$ with two additional relative size matched flat conditions (Fig. 3A). Results were consistent with our previous findings with smaller JND scores for apparent closer objects compared to all other depth conditions. Furthermore, no significant difference was found between the two flat conditions, allowing us to rule out surface edge proximity due to relative size differences as an alternative explanation (Fig. 3C).

The repeated measures ANOVA on the JND scores revealed a main effect of perceived depth $\left[\mathrm{F}_{(3,177)}=3.227, \mathrm{p}<.05, \eta_{\mathrm{p}}{ }^{2}=0.052\right]$. Notably, planned comparisons replicated previous results indicating smaller JND score for the close conditions (in accordance with the "close 
advantage" effect) compared to the far $\left[\mathrm{t}_{(59)}=2.133, \mathrm{p}<0.05\right.$; Mean Difference $=0.176, \mathrm{CI}$ :

$0.010,0.341]$, the flat-small $\left[\mathrm{t}_{(59)}=2.029, \mathrm{p}<0.05\right.$; Mean Difference $=0.192$, CI: $\left.0.002,0.381\right]$ and the flat-big condition $\left[\mathrm{t}_{(59)}=-3.368, \mathrm{p}<0.05\right.$; Mean Difference $=0.240$, CI: $\left.0.097,0.382\right]$. There was no significant difference between the far condition and the two flat conditions: far and flat-small $\left[\mathrm{t}_{(59)}<1\right.$; Mean Difference $\left.=-0.016, \mathrm{CI}:-0.195,0.163 ;\right]$, and far and flat-big $\left[\mathrm{t}_{(59)}<1\right.$ Mean Difference $=-0.064$, CI: $-0.202,0.075]$.

To examine whether edge proximity modulated JNDs, we employed a Bayesian paired samples t-test on the two flat conditions. In contrast to the Null Hypothesis Significance Testing, a Bayesian t-test can also provide evidence in favor of the null hypothesis (that is - no difference between the two flat conditions) (Van den Bergh et al., 2019; Wagenmakers et al., 2018). The Bayesian t-test supported the null hypothesis (i.e., no difference between the two flat conditions) $\left[\mathrm{BF}_{10}=0.164\right]$ such that the null hypothesis was 6.25 more likely than the H1 hypothesis. This result suggested that the surface edge proximity did not mediate the close advantage effect found in our previous studies.

The analysis of the RT did not produce significant results, $\left[\mathrm{F}_{(3,177)}<1\right]$. The lack of RT effect is consistent with previous literature that suggests that online behavioural studies may add noise to reaction times due to differences in hardware (de Leeuw \& Motz, 2015; Reimers \& Stewart, 2015). To conclude, the results of Experiment $2 \mathrm{~b}$ complemented those found in Experiment 2a (Fig. 2B), and further confirmed that the close advantage cannot be accounted for by the relative sizes of the surfaces. 
A

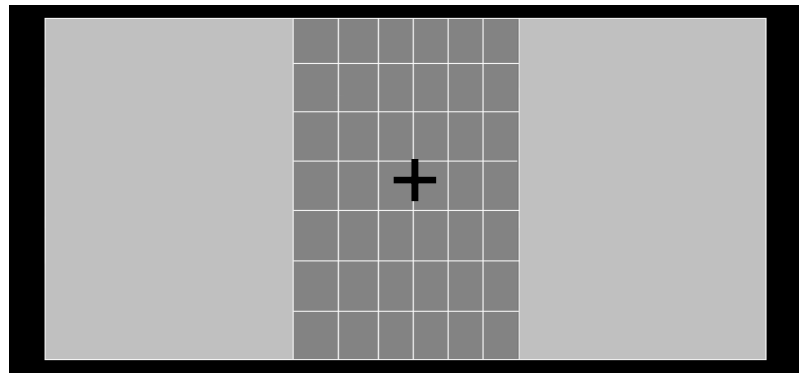

B

$\begin{array}{|lllccc|} & & & & & \end{array}$

C
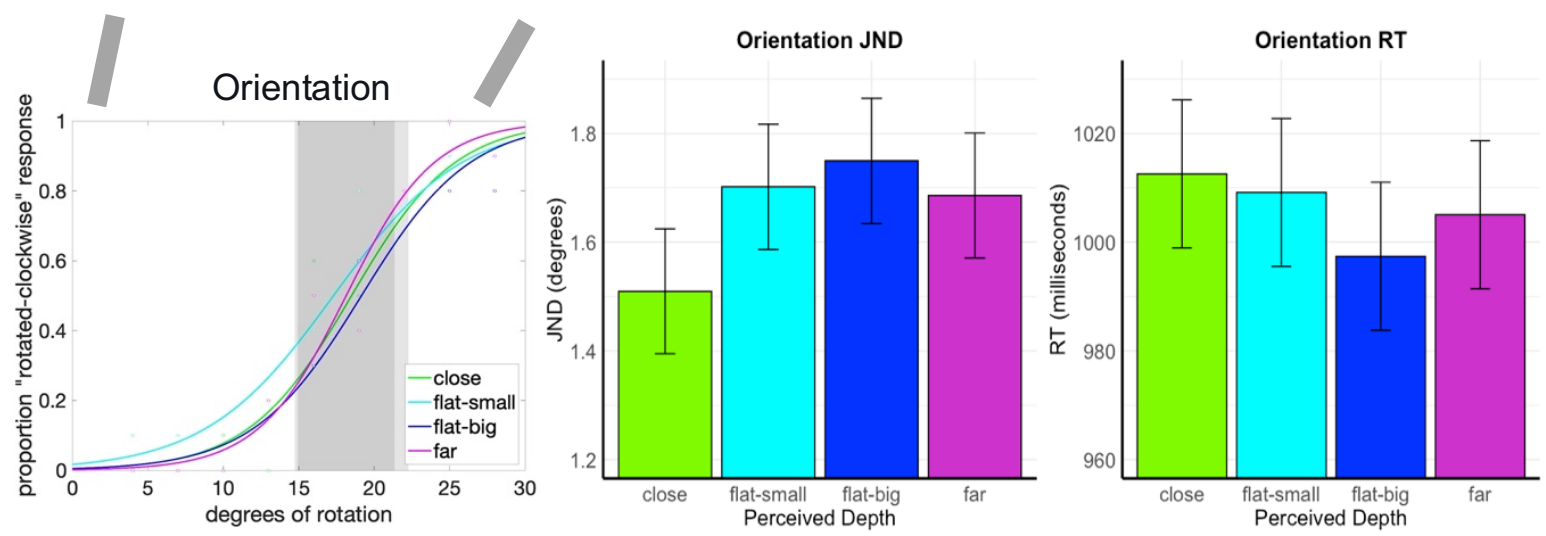

Fig. 3 Experiment 2b Stimuli and results A) Ponzo size matched Flat conditions - The left image (flat-small) is matched with the smaller rectangle (far condition) and the right image (flat-big) is matched with the bigger rectangle (close condition) of the Ponzo Illusion B) Experimental stimuli -lines of different orientations with target line changing with a step size of $3^{\circ}$, reference line at a constant orientation of $15^{\circ}$. C) Results - Left graph shows the psychometric fit for one participant, middle graph shows the average JNDs, and right graph shows average RTs. 


\section{Experiment 3: Face Identification}

The experiments described above examined the effects of perceived depth on the processing of low-level visual features. An outstanding question is whether the observed close advantage extends to higher level visual tasks, such as object recognition (Blini et al., 2018) and target identification (Li et al., 2011). Here, we focus on face perception, a high-level ability of the human visual system. A previous study already showed that participant's PPS was altered by the emotional status of a face; participants stopped angry avatars earlier (farther away from them) compared with happy avatars (Ruggiaro et al. 2017). The goal of Experiment 3 was to examine whether such subjective judgments also translate to psychophysical sensitivity. To this end, we examined whether face perception abilities are modulated by their perceived position in depth relative to an observer.

\section{Methods}

Participant

Data was collected from 32 participants (age: $\mathrm{M}=20.93, \mathrm{SD}=5.48 ; 5$ males). Sample size was based on that used in previous face perception studies (e.g., Hadad et al., 2019). Apriori we decided to recruit more participants compared with previous experiments given the known variability in face perception abilities (Bobak et al., 2016; Freud et al., 2020). One participant was excluded as their JNDs deviated from the average of the sample by more than four standard deviations. All other aspects of the recruiting process was the same as that described in Experiments 1 and 2a. 


\section{Materials and apparatus}

The size of the backgrounds (Ponzo Illusion and Ponzo Flat - See Fig. 1A) were doubled to subtend a visual angle of $54.61^{\circ} \times 27.63^{\circ}$ in order to accommodate the larger stimuli size. The stimulus set for Experiment 3 was obtained from a previous study (Hadad et al., 2019). The stimuli consisted of two base faces (unmorphed) that were morphed to form a continuum of faces (See Fig. 4A for sample). Faces were always presented at a visual angle of $6.81^{\circ} \times 8.05^{\circ}$. Face stimuli were controlled for luminance and contrast using the SHINE Toolbox in MATLAB (Willenbockel et al., 2010).

\section{Procedure}

Participants were given an opportunity at the beginning of the experiment to study the two unmorphed faces, labelled "Kyle" or "Fred". Then participants underwent eight supervised practice trials where they viewed morphed faces, presented one at a time, in the close, flat, or far portion of the Ponzo Illusion. They were asked to identify which face they saw by pressing the keys K (for Kyle) or F (for Fred). After ensuring participants understood the task correctly, they performed a brief pretest consisting of 24 trials repeated 4 times (total 96 trials) which was used to determine appropriate step size for the main experiment (but was not used in the final analysis). In the main experiment, they completed 11 levels, with step sizes ranging from $3 \%$ to $9 \%$ level of morphing, centered on the midpoint (50\% morphing). The main experiment consisted of a total of 66 trials ( 11 faces $\times 3$ depths $\times 2$ counterbalanced sides), repeated 12 times (total 792 trials), hence, each level was presented 22 times (11 faces x 2 counterbalanced sides) in a randomized order. 


\section{Results}

In this study, participants performed a face identification task to determine whether the "close advantage" is generalizable to higher-level visual tasks. Consistent with Experiments 1-2, faces placed on the close surface yielded smaller JNDs (more accurate classification) relative to the other conditions (Fig. 4B depicts the mean JND scores and RT values).

Repeated measures ANOVA of the JND scores found a main effect of perceived depth [ $\left.\mathrm{F}_{(2,60)}=3.683, \mathrm{p}<.05, \eta_{\mathrm{p}}^{2}=.109\right]$. Planned comparisons revealed a significantly lower JND score for faces perceived as closer in comparisons to the far condition: $(\mathrm{t}(30)=2.485, \mathrm{p}=0.01$, Mean Difference $=0.349,[\mathrm{CI}: 0.062,0.635])$, as well as the close and flat condition: $\left(\mathrm{t}_{(30)}=2.060, \mathrm{p}=\right.$ 0.048, Mean Difference $=0.326,[C I: 0.002,0.648])$. The far and flat condition did not show significant differences $\left(\mathrm{t}_{(30)}=0.174, \mathrm{p}=0.86\right.$; Mean Difference $=0.023$, [CI: $\left.\left.-0.245,0.291\right]\right)$. Together, the JND results supported the "close advantage" account and provided evidence of better perceptual resolution for faces that were perceived as closer.

Statistical analysis of the RT data did not reveal a significant main effect of perceived depth $\left[\mathrm{F}_{(2,60)}=2.632, \mathrm{p}=0.109 \eta_{\mathrm{p}}{ }^{2}=0.081^{2}\right]$. Importantly, however, there was no evidence for speed-accuracy trade-off as the trend observed for RT was consistent with that observed for the JND results.

\footnotetext{
2 Analysis was corrected for sphericity using the Greenhouse-Geisser method (to account for violation of the sphericity assumption)
} 
A

\begin{tabular}{|lrrrr|r|}
\hline Face (\% Kyle): & $0 \%$ & $25 \%$ & $50 \%$ & $75 \%$ & $100 \%$ \\
Face (\% Fred) & $100 \%$ & $75 \%$ & $50 \%$ & $25 \%$ & $0 \%$ \\
\hline
\end{tabular}

B
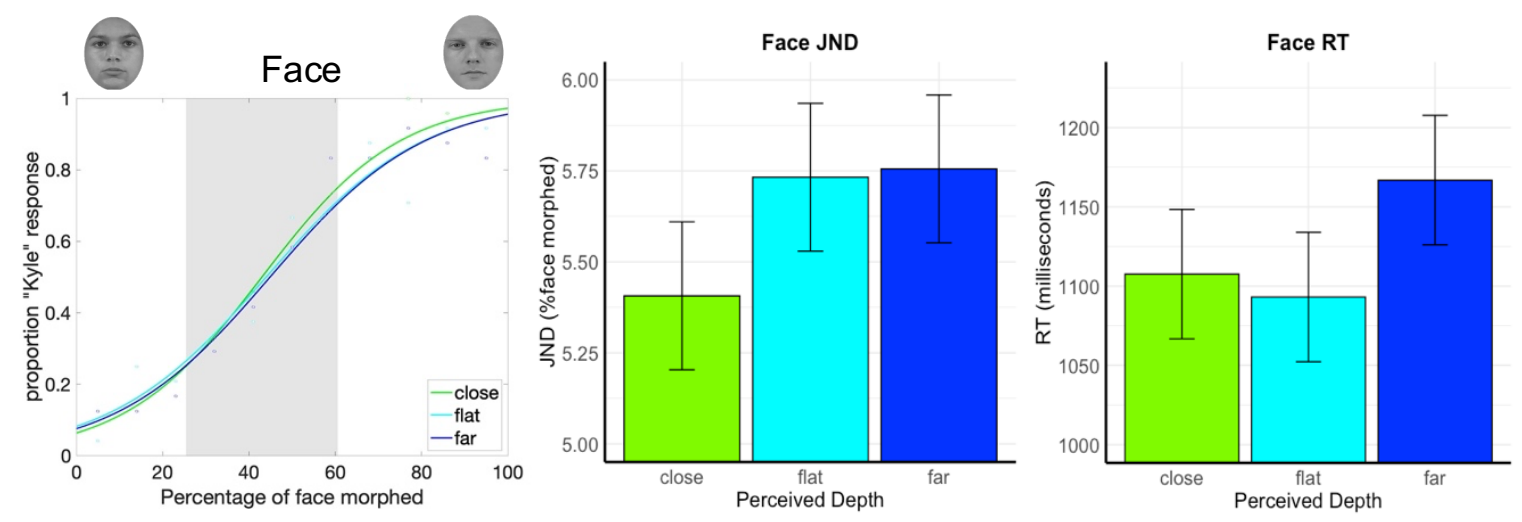

Fig. 4 Experiment 3 Stimuli and Results A) Experimental stimuli - sample faces presented in morphed in reference to the face "Kyle" and "Fred". Standard face is 50\%, halfway morphing between the two faces. B) Results: Left panel shows the psychometric fit curve for one participant, middle graph shows average JNDs, and the right graph shows average RTs. 


\section{Discussion}

The goal of the current study was to determine whether perceptual resolution is modulated by perceived depth. We assessed this across a variety of tasks and visual attributes by presenting stimuli on the 'close', 'flat', and 'far' version of Ponzo Illusion backgrounds. Our results reveal a consistent effect of depth, where more precise (lower JND) and faster processing was observed for objects positioned on the surface that appeared closer. This was evident for lower-level visual attributes such as size and orientation (Experiment 1, 2a, 2b) as well as a highlevel visual task, such as face perception (Experiment 3).

While we do not always find an effect of perceived distance on reaction times in our experiments, our findings are aligned with that of Blini et al. (2018), as both studies found a consistent advantage for objects that are perceived to be closer to the observer. Importantly, the current study extended those previous results along two critical dimensions. First, we generalized the close advantage effect from low-level to high-level visual tasks. Second, we demonstrated that the "close advantage" can be attributed to an enhancement in psychophysical sensitivity. While we cannot rule out that response bias contributes to the differences between the far and close condition, it is still the case that JND scores are more likely to reflect perceptual resolution rather than response bias.

Notably, previous research suggests that the close advantage effect is not evident across all tasks and conditions. In particular, a study by $\mathrm{Li}$ and colleagues (2011) reported a consistent advantage for detection of targets that were presented on a closer surface. However, in contrast to the current findings and to Blini et al.'s (2018) results, this early study did not find any advantage for identification of these close targets. The apparent inconsistencies between these studies might be explained by the nature of the task employed by Li et al. (2011), where 
participants were required to complete a dual task for two targets presented in the center and the periphery of the visual field. Additional research may be needed to characterize how the close advantage phenomenon is modulated by location across the visual field and by task demands.

What are the mechanisms that mediate the close advantage?

It is well established that depth perception modulates visual perception. This is best illustrated by Emmert's Law (Emmert, 1881); Emmert showed that an afterimage, which has a fixed retinal size, changes apparent size depending on the distance of a surface on which it is seen. If the observer looks at the afterimage on a near surface it appears smaller than if it is seen on a far surface. This relationship between perceived size and perceived distance was found to influence the activity of early visual cortex, such that objects that are perceived as bigger result in more neural activation in the early visual cortex (EVC) (Murray et al., 2006). Interestingly, these findings were used to predict a reverse pattern to that observed here. Specifically, according to this view, if objects on the far surface recruit more EVC processing, they should be seen with higher resolution than those perceived to be closer (and smaller). However, there is limited psychophysical support for this idea (Schindel \& Arnold, 2010; Lages et al., 2017), instead, there is more consistent evidence in favor of enhanced processing of close objects. Thus, the question regarding the neural mechanisms underlying the close advantage remains an open one.

One account attribute the close advantage to differential processing between the PPS and EPS (di Pellegrino \& Làdavas, 2015). For example, di Pellegrino \& Frassinetti (2000) provided evidence for privileged visual processing in the PPS compared with the EPS in patients with lesions to the parietal cortex. In particular, the authors found that visual extinction, a pathological 
bias of favouring recognition of objects presented to the ipsilesional visual field, was less evident for stimuli presented in the PPS. This finding is complemented by behavioural studies that show a faster rate of image processing (Reed et al., 2006), increased attentional prioritization, and slower attentional disengagement from tasks (Abrams et al., 2008), when objects were presented closer to the hand than farther away.

Importantly, in contrast to previous findings, our results show that the close advantage is evident even for perceived, rather than real, depth, and even when hand location was not manipulated. Hence, these results suggest that the close advantage occurs even when depth is solely defined by pictorial cues and therefore cannot be fully explained by the classic PPS/EPS account. Instead, the close advantage phenomenon might be better accounted for by affordance, the mere potential for action offered by objects (Bamford et al., 2020). For instance, a recent study by O'Connor et al., 2021 shows that spatial proximity to reward increases impulsive behaviour since objects of greater value (such as a food reward) can afford a more valuable outcome in closer proximity. Notably, and consistent with the current findings, previous studies have also demonstrated that affordance could influence behavior even when pictures, and not real objects, are used as experimental stimuli (e.g., Creem et al., 2001).

As such, objects that are strongly associated with actions (i.e., affordance), such as manmade tools that are behaviourally relevant may elicit a greater close advantage. Indeed, previous work has shown that even perceiving objects that potentiates action can alter behaviour. For instance, participants viewing pictures of objects with handles oriented towards their ipsilateral hand (i.e., easier to grasp) were faster to respond than when handles were orientated to the contralateral hand (Tucker \& Ellis, 1998; 2001; 2004). A recent study by Pilacinski et al. (2021) has shown that even eye saccades are primed for tool heads (the functional part of the tool), over 
tool handles. As such, an enhanced resolution of the feature-rich tool heads may help facilitate recognition of the tool's unique identity and functionality.

Moreover, affordance was not only modulated by object features that evoked actions, but also whether the object was within reachable space to act on. In one notable study by Witts et al. (2005), participants were asked to estimate distances to the target while holding or not holding a tool, with the intention of reaching the target or not. Although targets were always presented at the same distance away, participants perceived target to be closer when holding a tool, with the intention of using them. Linkenauger et al. (2009) corroborated these findings by showing that tools that are more difficult to pick up were perceived as farther than those easier to pick up.

Finally, another possible contributing factor to the close advantage phenomena could be attentional biases. Although we did not explicitly test this in our current study, it is well established that spatial attention is not uniformly distributed along the dimension of depth (Shelton et al., 1990, Gawryszewski et al. 1987; See Goodhew et al., 2015 for a review). Therefore, it is possible that closer object may receive a more dedicated attentional processing (Makin et al. 2009), which in turn facilitated the processing of these objects. This is in line with Li et al.'s (2011) findings that show differential processing at near and far distances as a result of attentional load, as well as other previous studies that support an attentional enhancement in the near space (Reed et al., 2006; Reed et al., 2013). Future studies should disentangle between perceptual and attentional processes that might contribute to the observed close advantage effect.

\section{"Close advantage" or "Far disadvantage"?}

An outstanding question is whether the difference observed between the close and far conditions in our study reflected facilitation of the close space processing ("close advantage"), 
interference in the processing of the far space ("far disadvantage"), or a combination of those two processes. To disentangle these options, we included the flat condition, for comparison, across the four experiments. We expected that performance in the flat condition would consistently fall midway between the close and far conditions, however this was not the case. Instead, in 3 of our 4 experiments (Experiments 2a, 2b, and 3) we found that performance in the flat condition was equivalent to that seen in the far condition with JNDs significantly larger than those obtained in the close condition. This supports the interpretation that the observed results are not due to a reduction in sensitivity as a function of distance, but instead reflect a sensitivity enhancement for the close surface. The results of Experiment 1 differ from the other experiments in that JNDs for the flat condition are statistically smaller than those found in the far condition. As outlined previously, it is possible that the relative performance across conditions in Experiment 1 was influenced by the interrelationship between size and perceived distance.

\section{Conclusion}

To conclude, the present series of experiments provide supportive evidence of higher perceptual resolution for objects that are perceived as closer to the observer. This benefit is seen consistently, across visual properties such as length and orientation, and even higher-level properties such as face identification. Together, our results point towards the existence of a dedicated processing mechanisms for closer things. 


\section{References}

Abrams, R. A., Davoli, C. C., Du, F., Knapp III, W. H., \& Paull, D. (2008). Altered vision near the hands. Cognition, 107(3), 1035-1047. https://doi.org/10.1016/j.cognition.2007.09.006

Baird, J. C., \& Noma, E. J. (1978). Fundamentals of scaling and psychophysics. John Wiley \& Sons.

Bamford, L. E., Klassen, N. R., \& Karl, J. M. (2020). Faster recognition of graspable targets defined by orientation in a visual search task. Experimental brain research, 238(4), 905916. https://doi.org/10.1007/s00221-020-05769-z

Bobak, A. K., Pampoulov, P., \& Bate, S. (2016). Detecting superior face recognition skills in a large sample of young British adults. Frontiers in psychology, 7, 1378. https://doi.org/10.3389/fpsyg.2016.01378

Blini, E., Desoche, C., Salemme, R., Kabil, A., Hadj-Bouziane, F., \& Farnè, A. (2018). Mind the depth: Visual perception of shapes is better in peripersonal space. Psychological science, 29(11), 1868-1877. https://doi.org/10.1177/0956797618795679

Canzoneri, E., Ubaldi, S., Rastelli, V., Finisguerra, A., Bassolino, M., \& Serino, A. (2013). Tooluse reshapes the boundaries of body and peripersonal space representations. Experimental brain research, 228(1), 25-42.

Costantini, M., Ambrosini, E., Tieri, G., Sinigaglia, C., \& Committeri, G. (2010). Where does an object trigger an action? An investigation about affordances in space. Experimental brain research, 207(1-2), 95-103. https://doi.org/10.1007/s00221-010-2435-8

Costantini, M., Ambrosini, E., Scorolli, C., \& Borghi, A. M. (2011). When objects are close to me: affordances in the peripersonal space. Psychonomic bulletin \& review, 18(2), 302-308. 
Creem, S. H., \& Proffitt, D. R. (2001). Grasping objects by their handles: a necessary interaction between cognition and action. Journal of experimental psychology: Human Perception and Performance, 27(1), 218. https://doi.org/10.1037/0096-1523.27.1.218

De Leeuw, J., \& Motz, B. (2015). Data \& experiment code for" Psychophysics in a web browser?".

Di Pellegrino, G., \& Frassinetti, F. (2000). Direct evidence from parietal extinction of enhancement of visual attention near a visible hand. Current Biology, 10(22), 1475-1477. https://doi.org/10.1016/S0960-9822(00)00809-5

Di Pellegrino, G., \& Làdavas, E. (2015). Peripersonal space in the brain. Neuropsychologia, 66, 126-133. https://doi.org/10.1016/j.neuropsychologia.2014.11.011

Dufour, A., \& Touzalin, P. (2008). Improved visual sensitivity in the perihand space. Experimental Brain Research, 190(1), 91-98. https://doi.org/10.1007/s00221-008$1453-2$

Emmert, E. (1881). Grossenverhaltnisse der nachbilder. Klinische Monatsblatter fur Augenheilkunde, 19, 443-450.

Francis, G., Cummins, B., Kim, J., Grzeczkowski, L., \& Thunell, E. (2019). The moon size illusion does not improve perceptual judgments. Consciousness and cognition, 73, 102754.

Freud, E., Stajduhar, A., Rosenbaum, R. S., Avidan, G., \& Ganel, T. (2020). The COVID-19 pandemic masks the way people perceive faces. Scientific reports, 10(1), 1-8. https://doi.org/10.1038/s41598-020-78986-9

Freud, E., Binur, N., Srikanth, A., Davidson, E., Ganel, T., \& Hadad, B. S. (2021). Double dissociation between perception and action in children. Journal of experimental child psychology, 201, 104986. https://doi.org/10.1016/j.jecp.2020.104986 
Gandhi, T., Kalia, A., Ganesh, S., \& Sinha, P. (2015). Immediate susceptibility to visual illusions after sight onset. Current Biology, 25(9), R358-R359.

https://doi.org/10.1016/j.cub.2015.03.005

Ganel, T., Tanzer, M., \& Goodale, M. A. (2008). A double dissociation between action and perception in the context of visual illusions: opposite effects of real and illusory size. Psychological Science, 19(3), 221-225. https://doi.org/10.1111/j.14679280.2008.02071.x

de Gonzaga Gawryszewski, L., Riggio, L., Rizzolatti, G., \& Umiltá, C. (1987). Movements of attention in the three spatial dimensions and the meaning of "neutral" cues. Neuropsychologia, 25(1), 19-29.

Goodhew, S. C., Edwards, M., Ferber, S., \& Pratt, J. (2015). Altered visual perception near the hands: A critical review of attentional and neurophysiological models. Neuroscience \& Biobehavioral Reviews, 55, 223-233.

Gunderson, V. M., Yonas, A., Sargent, P. L., \& Grant-Webster, K. S. (1993). Infant macaque monkeys respond to pictorial depth. Psychological Science, 4(2), 93-98. https://doi.org/10.1111/j.1467-9280.1993.tb00467.x

Hadad, B. S., Schwartz, S., \& Binur, N. (2019). Reduced perceptual specialization in autism: Evidence from the other-race face effect. Journal of Experimental Psychology: General, 148(3), 588. http://dx.doi.org/10.1037/xge0000550

Howard, I. P. (2012). Perceiving in depth, volume 1: basic mechanisms. Oxford University Press. 
Kaas, A. L., \& Van Mier, H. I. (2006). Haptic spatial matching in near peripersonal space. Experimental Brain Research, 170(3), 403-413. https://doi.org/ 10.1007/s00221005-0223-7

Kaufman, L., \& Kaufman, J. H. (2000). Explaining the moon illusion. Proceedings of the National Academy of Sciences, 97(1), 500-505.

Làdavas, E. (2002). Functional and dynamic properties of visual peripersonal space. Trends in cognitive sciences, 6(1), 17-22. https://doi.org/10.1016/S1364-6613(00)01814-3

Lages, M., Boyle, S. C., \& Jenkins, R. (2017). Illusory increases in font size improve letter recognition. Psychological science, 28(8), 1180-1188.

Li, T., Watter, S., \& Sun, H. J. (2011). Differential visual processing for equivalent retinal information from near versus far space. Neuropsychologia, 49(14), 3863-3869. https://doi.org/10.1016/j.neuropsychologia.2011.10.002

Linkenauger, S. A., Witt, J. K., Stefanucci, J. K., Bakdash, J. Z., \& Proffitt, D. R. (2009). The effects of handedness and reachability on perceived distance. Journal of Experimental Psychology: Human Perception and Performance, 35(6), 1649.

Makin, T. R., Holmes, N. P., Brozzoli, C., Rossetti, Y., \& Farne, A. (2009). Coding of visual space during motor preparation: approaching objects rapidly modulate corticospinal excitability in hand-centered coordinates. Journal of Neuroscience, 29(38), 11841-11851.

Mason, W., \& Suri, S. (2012). Conducting behavioral research on Amazon’s Mechanical Turk. Behavior research methods, 44(1), 1-23. https://doi.org/10.3758/s13428-011-0124-6 Murray, S. O., Boyaci, H., \& Kersten, D. (2006). The representation of perceived angular size in human primary visual cortex. Nature neuroscience, 9(3), 429-434. 
O'Connor, D. A., Janet, R., Guigon, V., Belle, A., Vincent, B. T., Bromberg, U., ... \& Dreher, J. C. (2021). Rewards that are near increase impulsive action. Iscience, 24(4), 102292.

Palan, S., \& Schitter, C. (2018). Prolific. ac-A subject pool for online experiments. Journal of Behavioral and Experimental Finance, 17, 22-27. https://doi.org/10.1016/j.jbef.2017.12.004

Peirce, J. W. (2007). PsychoPy—psychophysics software in Python. Journal of neuroscience methods, 162(1-2), 8-13. https://doi.org/10.1016/j.jneumeth.2006.11.017

Peirce, J., Gray, J. R., Simpson, S., MacAskill, M., Höchenberger, R., Sogo, H., ... \& Lindeløv, J. K. (2019). PsychoPy2: Experiments in behavior made easy. Behavior research methods, 51(1), 195-203. https://doi.org/10.3758/s13428-018-01193-y

Pilacinski, A., De Haan, S., Donato, R., \& Almeida, J. (2021). Tool heads prime saccades. Scientific reports, $11(1), 1-8$.

Previc, F. H. (1990). Functional specialization in the lower and upper visual fields in humans: Its ecological origins and neurophysiological implications. Behavioral and Brain Sciences, 13(3), 519-542. doi:10.1017/S0140525X00080018

Previc, F. H. (1998). The neuropsychology of 3-D space. Psychological bulletin, 124(2), 123. https://doi.org/10.1037/0033-2909.124.2.123

Jarmasz, J., \& Hollands, J. G. (2009). Confidence intervals in repeated-measures designs: The number of observations principle. Canadian Journal of Experimental Psychology, 63(2), 124.

JASP Team (2018). JASP (Version 0.8.5) [Computer software]. JASP Team.

R Core Team (2020). R: A language and environment for statistical computing. R Foundation for Statistical Computing, Vienna, Austria. URL https:/www.R-project.org/. 
Reed, C. L., Grubb, J. D., \& Steele, C. (2006). Hands up: attentional prioritization of space near the hand. Journal of Experimental Psychology: Human Perception and Performance, 32(1), 166-177. https://doi.org/10.1037/0096-1523.32.1.166

Reed, C. L., Leland, D. S., Brekke, B., \& Hartley, A. A. (2013). Attention's grasp: Early and late hand proximity effects on visual evoked potentials. Frontiers in Psychology, 4, 420.

Reimers, S., \& Stewart, N. (2015). Presentation and response timing accuracy in Adobe Flash and HTML5/JavaScript Web experiments. Behavior research methods, 47(2), 309-327. https://doi.org/10.3758/s13428-014-0471-1

Reips, U. D. (2000). The Web experiment method: Advantages, disadvantages, and solutions. Psychological experiments on the Internet, 89-117. https://doi.org/10.1016/B978012099980-4/50005-8

Rizzolatti, G., Fadiga, L., Fogassi, L., \& Gallese, V. (1997). The space around us. Science, 277(5323), 190-191. https://doi.org/10.1126/science.277.5323.190

Ruggiero, G., Frassinetti, F., Coello, Y., Rapuano, M., Di Cola, A. S., \& Iachini, T. (2017). The effect of facial expressions on peripersonal and interpersonal spaces. Psychological research, 81(6), 1232-1240.

Schindel, R., \& Arnold, D. H. (2010). Visual sensitivity can scale with illusory size changes. Current Biology, 20(9), 841-844.

Shelton, P. A., Bowers, D., \& Heilman, K. M. (1990). Peripersonal and vertical neglect. Brain, 113(1), 191-205. 
Tucker, M., \& Ellis, R. (1998). On the relations between seen objects and components of potential actions. Journal of Experimental Psychology: Human perception and performance, 24(3), 830 .

Tucker, M., \& Ellis, R. (2001). The potentiation of grasp types during visual object categorization. Visual cognition, 8(6), 769-800.

Tucker, M., \& Ellis, R. (2004). Action priming by briefly presented objects. Acta psychologica, 116(2), 185-203.

Tseng, P., \& Bridgeman, B. (2011). Improved change detection with nearby hands. Experimental brain research, 209(2), 257-269.

Timney, B., \& Keil, K. (1996). Horses are sensitive to pictorial depth cues. Perception, 25(9), 1121-1128. https://doi.org/10.1068/p251121

Wagenmakers, E. J., Love, J., Marsman, M., Jamil, T., Ly, A., Verhagen, J., ... \& Morey, R. D. (2018). Bayesian inference for psychology. Part II: Example applications with JASP. Psychonomic bulletin \& review, 25(1), 58-76. https://doi.org/10.3758/s13423-017$1323-7$

Willenbockel, V., Sadr, J., Fiset, D., Horne, G. O., Gosselin, F., \& Tanaka, J. W. (2010). Controlling low-level image properties: the SHINE toolbox. Behavior research methods, 42(3), 671-684. https://doi.org/10.3758/BRM.42.3.671

Witt, J. K., Proffitt, D. R., \& Epstein, W. (2005). Tool use affects perceived distance, but only when you intend to use it. Journal of experimental psychology: Human perception and performance, $31(5), 880$.

Urban, F. M. (1910). The method of constant stimuli and its generalizations. Psychological Review, 17(4), 229. 
Van den Bergh, D., van Doorn, J., Draws, T., van Kesteren, E. J., Derks, K., Dablander, F., ... \& Wagenmakers, E. J. (2019). A Tutorial on Conducting and Interpreting a Bayesian ANOVA in JASP. https://doi.org/10.31234/osf.io/spreb 